\title{
Instabilities of one-dimensional trivial-phase solutions of the two-dimensional cubic nonlinear Schrödinger equation
}

\author{
John D. Carter \\ Seattle University \\ Mathematics Department \\ Seattle, WA 98122-1090 \\ Bernard Deconinck \\ University of Washington \\ Department of Applied Mathematics \\ Seattle, WA 98195-2420
}

\begin{abstract}
The two-dimensional cubic nonlinear Schrödinger equation (NLS) is used as a model of a wide variety of physical phenomena. In this paper, we study the stability of a class of its one-dimensional, periodic, traveling-wave solutions. First, we establish that all such solutions are unstable with respect to two-dimensional perturbations with long wavelengths in the transverse dimension. Second, we establish that all such solutions are unstable with respect to two-dimensional perturbations with arbitrarily short wavelengths if the coefficients of the linear dispersion terms in NLS have opposite signs. Both arguments rely on formal perturbation methods. Third, we use the Fourier-Floquet-Hill numerical method to examine the spectral stability problem. We present detailed spectra for twelve different solutions and demonstrate strong agreement between the numerical and asymptotic results.
\end{abstract}

Key words: stability, nonlinear Schrödinger equation, NLS, periodic solutions, traveling waves

PACS: 0.23.Jr, 02.70.Hm, 42.65.Sf, 03.75.Nt, 92.10.Hm, 52.35.Mw, 92.10.Dh

Email addresses: carterj1@seattleu.edu (John D. Carter), bernard@amath. washington.edu (Bernard Deconinck). 


\section{Introduction}

The two-dimensional cubic nonlinear Schrödinger equation (NLS) is given by

$$
i \psi_{t}+\alpha \psi_{x x}+\beta \psi_{y y}+2|\psi|^{2} \psi=0
$$

where $\alpha$ and $\beta$ are real constants and $\psi=\psi(x, y, t)$ is a complex-valued function. Among many other physical situations, NLS arises as an approximate model of the evolution of a nearly monochromatic wave of small amplitude in pulse propagation along optical fibers $(\alpha \beta>0)[18]$, in gravity waves on deep water $(\alpha \beta<0)[2,5]$ and in Langmuir waves in a plasma $(\alpha \beta>0)[20]$. As a description of a Bose-Einstein condensate, NLS is known as the GrossPitaevskii equation $(\alpha \beta>0)[8,21]$. All quantities in (1) are dimensionless.

NLS admits a large class of one-dimensional traveling-wave solutions of the form

$$
\psi(x, t)=\phi(x) \mathrm{e}^{i \lambda t}
$$

where $\phi$ is a real-valued function and $\lambda$ is a real parameter. Solutions of the form given in (2) are known as one-dimensional trivial-phase solutions.

Remark: The most general form of a one-dimensional trivial-phase solution is given by

$$
\psi(x, y, t)=\phi(a x+b y-s t) \mathrm{e}^{i \lambda t+i a \bar{s} x+i b \bar{s} y+i \eta},
$$

where $\phi$ is a real-valued function, $\bar{s}=s /\left(2 \alpha a^{2}+2 \beta b^{2}\right)$ and $a, b, s, \lambda$ and $\eta$ are real parameters. By making use of the symmetries of NLS [23] and by redefining $\lambda$, all solutions of this form can be considered by studying the simplified form given in (2).

If $\alpha>0$, then NLS is said to be focusing (attractive) in the $x$-dimension and it admits the two trivial-phase solutions

$$
\begin{aligned}
& \phi(x)=\sqrt{\alpha} k \operatorname{cn}(x, k), \quad \text { with } \lambda=\alpha\left(2 k^{2}-1\right), \\
& \phi(x)=\sqrt{\alpha} \operatorname{dn}(x, k), \quad \text { with } \lambda=\alpha\left(2-k^{2}\right) .
\end{aligned}
$$

If $k=0$ in (5), then $\phi$ is constant and the corresponding $\psi$ is known as a Stokes' wave (plane wave) solution of NLS with amplitude $\sqrt{\alpha}$.

On the other hand, if $\alpha<0$, then NLS is said to be defocusing (repulsive) in the $x$-dimension and it admits the trivial-phase solution

$$
\phi(x)=\sqrt{-\alpha} k \operatorname{sn}(x, k), \quad \text { with } \lambda=-\alpha\left(1+k^{2}\right) .
$$

In (4)-(6), $k \in[0,1]$ is a free parameter known as the elliptic modulus and $\operatorname{cn}(\cdot, k), \operatorname{dn}(\cdot, k)$, and $\operatorname{sn}(\cdot, k)$ are Jacobi elliptic functions [3]. If $k<1$, then $\phi$ is periodic in $x$. The period of the $\phi$ 's given in (4) and (6) is $4 K(k)$ and 
the period of the $\phi$ given in $(5)$ is $2 K(k)$. Here $K(k)$ is the complete elliptic integral of the first kind [3] defined by

$$
K(k)=\int_{0}^{\pi / 2} \frac{1}{\sqrt{1-k^{2} \sin ^{2}(\theta)}} d \theta .
$$

Although this paper focuses on spatially-periodic trivial-phase solutions (i.e. solutions of the form given in (4), (5) or (6) with $k<1$ ), it should be noted that as $k \rightarrow 1$, the period of $\phi$ increases without bound and $\phi$ limits to an appropriate hyperbolic function. The resulting NLS solution $\psi$ is often called a solitary wave.

In this paper we investigate the linear stability of the solutions (4)-(6) in the context of (1). This gives rise to a spectral problem that we investigate perturbatively and numerically. We resort to these approximate methods because the spectra of the associated operators are not known. This is true even for the one-dimensional $(\rho=0)$ setting, with the exception of the soliton $(k=1)$ limit. Others have considered this problem, using a variety of approximate methods.

Davey \& Stewartson [5] established that all Stokes' wave solutions are unstable unless either $\alpha \beta=0$, or $\alpha<0$ and $\beta<0$. Zakharov \& Rubenchik [24] established that the solutions given in (4) and (5) with $k=1$ are unstable with respect to long-wavelength transverse perturbations regardless of the sign of $\beta$. Extensive reviews of the stability of solitary wave solutions are given in $[16,22,15,7]$. Martin et al. [19] numerically examined the stability of the spatially periodic solutions given in (5) for a range of $k$ values. They established that all such solutions are unstable with respect to two-dimensional perturbations. Their numerical results are a subset of the results we present in Section 4. Infeld \& Ziemkiewicz [10] established, using perturbation analysis, that all one-dimensional trivial-phase solutions of (1) are unstable with respect to two-dimensional perturbations that have long wavelengths in the transverse dimension. Carter \& Segur [4] summarized the asymptotic results we present in Section 3 and include a numerical result on the stability of one solution of the form (6). Their numerical method is different from the one used in this paper. A comparison of these two methods is given in Section 4. A method similar to the one used by Carter \& Segur [4] was used by Aleshkevich et al. [1] and Kartashov et al. $[12,13]$. Kartashov et al. [12] established that solutions of the form (4)-(5) are unstable with respect to one-dimensional perturbations, and that solution of the form (6) are stable with respect to one-dimensional perturbations. They also established that instabilities of (4) have complex growth rates. Their one-dimensional stability computations are a subset of the two-dimensional results presented in Section 4. Aleshkevich et al. [1] and Kartashov et al. [13] numerically examined the stability of the solutions given in (4)-(6). They established that all such solutions are unstable with respect 
to two-dimensional perturbations.

Our asymptotic results for long-wavelength transverse perturbations generalize the work of Zakharov \& Rubenchik [24]. Similar results are also found in Infeld \& Ziemkiewicz [10], but only for small $k$. Our results are valid for all $k \in[0,1]$. Our asymptotic results for short-wavelength transverse perturbations expands on the work by Carter \& Segur [4]. Our presentation of both the long- and short-wavelength results elaborates on that in [4] significantly. Any of the numerical results obtained by these authors are a subset of the numerical results presented in Section 4. This is due to the larger class of perturbations incorporated by our method. We do not impose any restrictions on the spatial period in $x$ of the perturbation modes.

\section{The linear stability problem}

In order to study the stability of solutions of the form given in (2), we consider perturbed solutions, $\psi_{p}=\psi_{p}(x, y, t)$, with the following structure

$$
\psi_{p}=\left(\phi(x)+\epsilon u(x, y, t)+i \epsilon v(x, y, t)+\mathcal{O}\left(\epsilon^{2}\right)\right) \mathrm{e}^{i \lambda t},
$$

where $u(x, y, t)$ and $v(x, y, t)$ are real-valued functions, $\epsilon$ is a small real parameter, and $\phi(x) \mathrm{e}^{i \lambda t}$ is one of the solutions presented in the previous section. Substituting (8) into (1), linearizing and separating into real and imaginary parts gives

$$
\begin{aligned}
-\lambda v+2 \phi^{2} v+\beta v_{y y}+\alpha v_{x x} & =-u_{t} \\
-\lambda u+6 \phi^{2} u+\beta u_{y y}+\alpha u_{x x} & =v_{t}
\end{aligned}
$$

Since the system given in (9) does not depend on $y$ or $t$ explicitly, we assume that $u(x, y, t)$ and $v(x, y, t)$ have the forms

$$
\begin{aligned}
& u(x, y, t)=U(x, \rho) \mathrm{e}^{i \rho y+\Omega t}+c . c ., \\
& v(x, y, t)=V(x, \rho) \mathrm{e}^{i \rho y+\Omega t}+c . c .
\end{aligned}
$$

where $\rho$ is a real constant, $\Omega$ is a complex constant, $U$ and $V$ are complexvalued functions and c.c. denotes complex conjugate. This leads to

$$
\begin{aligned}
& -\lambda V+2 \phi^{2} V-\beta \rho^{2} V+\alpha V_{x x}=-\Omega U, \\
& -\lambda U+6 \phi^{2} U-\beta \rho^{2} U+\alpha U_{x x}=\Omega V .
\end{aligned}
$$

This system can be rewritten as

$$
\left(\begin{array}{cc}
0 & -L_{-} \\
L_{+} & 0
\end{array}\right)\left(\begin{array}{l}
U \\
V
\end{array}\right)=\Omega\left(\begin{array}{l}
U \\
V
\end{array}\right)
$$


where

$$
\begin{aligned}
& L_{-}=-\lambda+2 \phi^{2}-\beta \rho^{2}+\alpha \partial_{x}^{2}, \\
& L_{+}=-\lambda+6 \phi^{2}-\beta \rho^{2}+\alpha \partial_{x}^{2} .
\end{aligned}
$$

Instability occurs if (11) admits a bounded solution with $\Re(\Omega)>0$. (The notation $\Re(\Omega)$ represents the real part of $\Omega$.) We refer to instabilities due to non-real $\Omega$ as oscillatory instabilities. We define the growth rate of an instability to be the real part of $\Omega$. The system (11) is the focus of this paper.

\section{$3 \quad$ Formal perturbation theory}

In this section formal perturbation techniques are used to obtain asymptotic estimates on parts of the spectrum of (11) for varying $\rho$. It may be possible for these techniques to be rigorously justified, but no attempts in that direction are made here.

\subsection{Long-wavelength instabilities}

Generalizing the work in [24], we assume that for fixed $k$ and for fixed small $\rho$ (long-wavelength transverse perturbations), (11) admits solutions of the form

$$
\begin{aligned}
U(x, \rho) & \sim u_{0}(x)+\rho u_{1}(x)+\rho^{2} u_{2}(x)+\ldots, \\
V(x, \rho) & \sim v_{0}(x)+\rho v_{1}(x)+\rho^{2} v_{2}(x)+\ldots, \\
\Omega & \sim \rho \omega_{1}+\rho^{2} \omega_{2}+\ldots,
\end{aligned}
$$

where the $\omega_{j}$ are complex constants and the $u_{j}$ and $v_{j}$ are complex-valued bounded functions.

Substituting (14) into (11) and separating by powers of $\rho$ gives

$$
\begin{aligned}
& -\lambda v_{0}+2 \phi^{2} v_{0}+\alpha v_{0 x x}=0, \\
& -\lambda u_{0}+6 \phi^{2} u_{0}+\alpha u_{0 x x}=0
\end{aligned}
$$

at leading order in $\rho, \mathcal{O}\left(\rho^{0}\right)$. The general solution to this system is

$$
\begin{aligned}
& u_{0}(x)=c_{1} \phi_{x}+c_{2} \phi_{x} \int_{0}^{x} \phi_{z}^{-2} d z \\
& v_{0}(x)=c_{3} \phi+c_{4} \phi \int_{0}^{x} \phi^{-2} d z
\end{aligned}
$$

where $c_{1}, c_{2}, c_{3}$ and $c_{4}$ are complex constants. The restriction that $u_{0}$ and $v_{0}$ must be bounded leads to the requirement that $c_{2}=c_{4}=0$ because both of 
the integral terms in (16) grow linearly with $x$. At the next order in $\rho, \mathcal{O}(\rho)$, (11) gives

$$
\begin{aligned}
-\lambda v_{1}+2 \phi^{2} v_{1}+\alpha v_{1 x x} & =-\omega_{1} u_{0} \\
-\lambda u_{1}+6 \phi^{2} u_{1}+\alpha u_{1 x x} & =\omega_{1} v_{0} .
\end{aligned}
$$

The general solution of this system is

$$
\begin{aligned}
& u_{1}(x)=c_{5} \phi_{x}+c_{6} \phi_{x} \int_{0}^{x} \phi_{z}^{-2} d z+\frac{\omega_{1} c_{3}}{2 \alpha} \phi_{x} \int_{0}^{x} \phi^{2} \phi_{z}^{-2} d z \\
& v_{1}(x)=c_{7} \phi+c_{8} \phi \int_{0}^{x} \phi^{-2} d z-\frac{\omega_{1} c_{1}}{2 \alpha} x \phi
\end{aligned}
$$

where $c_{5}, c_{6}, c_{7}$ and $c_{8}$ are complex constants. In order to ensure that $u_{1}$ and $v_{1}$ are bounded, $c_{6}$ and $c_{8}$ must be chosen to cancel all linear growth that results from the particular solution of the nonhomogeneous problem. Specifically,

$$
\begin{aligned}
& c_{6}=-\omega_{1} c_{3} \operatorname{sgn}(\alpha) \frac{f_{1}}{2 f_{2}}, \\
& c_{8}=\omega_{1} c_{1} \operatorname{sgn}(\alpha) \frac{1}{2 f_{3}} .
\end{aligned}
$$

Here and in what follows, the $f_{j}=f_{j}(k)$ are functions that depend on the parameter $k$ only. Analytic expressions for the $f_{j}$ 's corresponding to the solutions given in (4)-(6) are included in Appendices A.1-A.3.

At $\mathcal{O}\left(\rho^{2}\right),(11)$ gives

$$
\begin{aligned}
-\lambda v_{2}+2 \phi^{2} v_{2}+\alpha v_{2 x x} & =\beta v_{0}-\omega_{2} u_{0}-\omega_{1} u_{1}, \\
-\lambda u_{2}+6 \phi^{2} u_{2}+\alpha u_{2 x x} & =\beta u_{0}+\omega_{2} v_{0}+\omega_{1} v_{1} .
\end{aligned}
$$

The solution to this system has terms that grow linearly and others that grow quadratically. Ensuring that the right-hand sides of these equations are orthogonal to the periodic solutions of the homogeneous problems leads to the following pair of restrictions

$$
\begin{aligned}
& \left(\frac{\beta}{\alpha} f_{6}-\frac{\omega_{1}^{2}}{4 \alpha^{2}}\left(\frac{1}{f_{3}}-f_{5}\right)\right) c_{1}=0 \\
& \left(\frac{\beta}{\alpha} f_{5}-\frac{\omega_{1}^{2}}{4 \alpha^{2}}\left(\frac{f_{1}^{2}}{f_{2}}-f_{4}\right)\right) c_{3}=0 .
\end{aligned}
$$

Equation (21) admits two nontrivial solutions

$$
\begin{aligned}
& \omega_{1}^{2}=-4 \alpha \beta \frac{f_{3} f_{6}}{f_{3} f_{5}-1}, \quad c_{3}=0, \quad \text { and } \quad c_{1} \text { arbitrary }, \\
& \omega_{1}^{2}=-4 \alpha \beta \frac{f_{2} f_{5}}{f_{2} f_{4}-f_{1}^{2}}, \quad c_{1}=0, \quad \text { and } \quad c_{3} \text { arbitrary } .
\end{aligned}
$$


To leading order, (22a) leads to the "snake" mode

$$
\begin{gathered}
U_{s}(x, \rho)=\phi_{x}, \\
V_{s}(x, \rho)=0, \\
\Omega_{s}^{2}=-4 \alpha \beta \rho^{2} \frac{f_{3} f_{6}}{f_{3} f_{5}-1}=-\alpha \beta \rho^{2} \omega_{1 s},
\end{gathered}
$$

and (22b) leads to the "neck" mode

$$
\begin{gathered}
U_{n}(x, \rho)=0 \\
V_{n}(x, \rho)=\phi \\
\Omega_{n}^{2}=-4 \alpha \beta \rho^{2} \frac{f_{2} f_{5}}{f_{2} f_{4}-f_{1}^{2}}=-\alpha \beta \rho^{2} \omega_{1 n},
\end{gathered}
$$

where $\omega_{1 s}$ and $\omega_{1 n}$ are functions that depend only on $k$. These results are presented in Figure 1, where the scaled growth rates, $\omega_{1 s}$ and $\omega_{1 n}$ are plotted versus $k$ for the unperturbed solutions given in (4)-(6). These plots show that $\omega_{1 n}<0$ for (4) and (5) and $\omega_{1 s}<0$ for (6). Therefore, if $\alpha \beta>0$, (4) and (5) are unstable with respect to long-wave transverse perturbations corresponding to the neck mode and (6) is unstable with respect to long-wave transverse perturbations corresponding to the snake mode. These plots also show that $\omega_{1 s}>0$ for (4) and (5) and $\omega_{1 n}>0$ for (6). Therefore, if $\alpha \beta<0$, (4) and (5) are unstable with respect to the snake mode and (6) is unstable with respect to the neck mode. These asymptotic results are corroborated numerically in Section 4 .

A "snaking" instability has the opposite parity in $x$ from the unperturbed solution and causes the crests and troughs to oscillate forward and backward. A "necking" instability has the same parity in $x$ as the unperturbed solution and causes the crests to alternate between being high and narrow and low and broad.

\section{Remarks:}

- This asymptotic argument establishes that all one-dimensional trivial-phase solutions with $0 \leq k<1$ are unstable with respect to two-dimensional perturbations with long wavelengths in the transverse dimension regardless of the signs of $\alpha$ and $\beta$.

- A one-dimensional trivial-phase solution of NLS is unstable to a growing neck mode if $\beta>0$, and to a growing snake mode if $\beta<0$.

- Although the transverse wavelength of the perturbation is large, the longitudinal scale of the perturbation is the same as the scale of the unperturbed solution.

- For all values of $k$, in the small- $\rho$ limit, the scaled growth rates are finite. Specifically, $0 \leq\left|\omega_{1}\right| \leq 12$. 
(a)

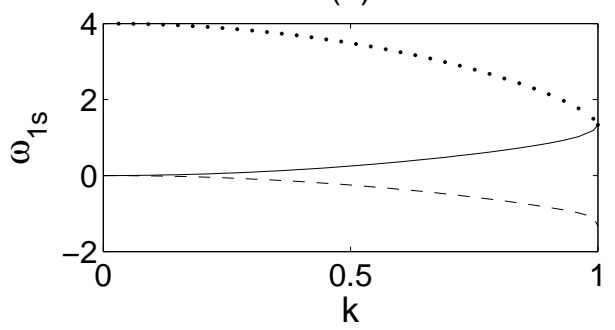

(b)

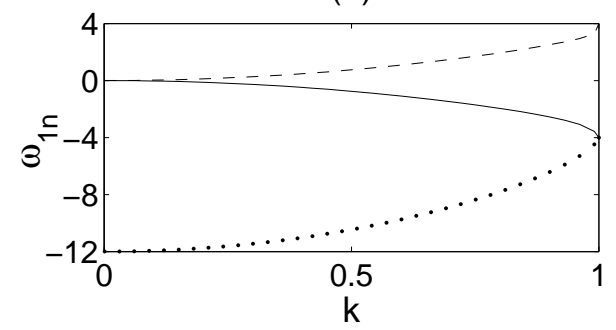

Fig. 1. Plots of $\omega_{1 s}$ and $\omega_{1 n}$ versus $k$ in (a) and (b) respectively. The solid lines correspond to (4), the dotted lines correspond to (5) and the dashed lines correspond to $(6)$.

\subsection{Short-wavelength instabilities}

If $\alpha \beta>0$, the last two terms on the left-hand sides of (11a) and (11b) have the same sign because the second derivative is a negative operator. Additionally, if $\rho$ is large, then these two terms dominate the other left-hand side terms. If $\rho$ is chosen large enough to satisfy

$$
\rho^{2}>5\left|\frac{\alpha}{\beta}\right|
$$

then this dominance is established and the two operators on the left-hand side of (11) have the same sign. Multiply (11a) and (11b) through by $U$ and $V$ respectively and integrate both equations with respect to $x$ over one period of the unperturbed solution. If the left-hand sides of the resulting equations are multiplied, their product is positive because the operators have the same sign (recall that $U$ and $V$ are real functions). The product of the right-hand sides results in $-\Omega^{2}$ times the square of the integral of $U V$. Therefore, $\Omega^{2}<0$ at leading order in $\rho$ and there is no large- $\rho$ instability if $\alpha \beta>0$. This result is corroborated numerically in Section 4.

For fixed $k, \alpha \beta<0$, and fixed large $\rho$ (short-wavelength transverse perturbations), assume

$$
\begin{aligned}
U(x) & \sim \zeta_{0}(z)+\rho^{-2} \zeta_{1}(z)+\rho^{-4} \zeta_{2}(z)+\ldots, \\
V(x) & \sim \xi_{0}(z)+\rho^{-2} \xi_{1}(z)+\rho^{-4} \xi_{2}(z)+\ldots, \\
\Omega & \sim w_{0}+\rho^{-2} w_{1}+\rho^{-4} w_{2}+\ldots, \\
\alpha \mu^{2} & \sim-\beta \rho^{2}+\mu_{0}+\rho^{-2} \mu_{1}+\ldots,
\end{aligned}
$$

where $z=\mu x$, the $w_{j}$ are complex constants, the $\mu_{j}$ are real constants and the $\zeta_{j}$ and $\xi_{j}$ are complex-valued bounded functions. The wave number $\mu$ is real only if $\alpha \beta<0$. This is the only case we consider in this section. Note that if $\rho$ is large, then $\mu$ is necessarily large as well. 
Substituting (26) into (11), simplifying and separating by powers of $\rho$ leads to

$$
\begin{aligned}
& \zeta_{0 z z}+\zeta_{0}=0, \\
& \xi_{0 z z}+\xi_{0}=0,
\end{aligned}
$$

at leading order in $\rho, \mathcal{O}\left(\rho^{2}\right)$. The general solution of this system is

$$
\begin{aligned}
& \zeta_{0}(z)=b_{1} \sin (z)+b_{2} \cos (z), \\
& \xi_{0}(z)=b_{3} \sin (z)+b_{4} \cos (z),
\end{aligned}
$$

where $b_{1}, b_{2}, b_{3}$ and $b_{4}$ are complex constants. At the next order in $\rho, \mathcal{O}\left(\rho^{0}\right)$, (11) gives

$$
\begin{aligned}
& \beta \xi_{1 z z}+\beta \xi_{1}=w_{0} \zeta_{0}-\lambda \xi_{0}+2 \phi^{2} \xi_{0}+\mu_{0} \xi_{0 z z}, \\
& \beta \zeta_{1 z z}+\beta \zeta_{1}=-w_{0} \xi_{0}-\lambda \zeta_{0}+6 \phi^{2} \zeta_{0}+\mu_{0} \zeta_{0 z z} .
\end{aligned}
$$

In order for $\zeta_{1}$ and $\xi_{1}$ to be bounded, the right-hand side of (29) must be orthogonal to the solutions of the corresponding homogeneous problem. This requirement leads to the restrictions

$$
\left(\begin{array}{cccc}
w_{0} & 0 & -\lambda+2 g_{1}-\mu_{0} & 0 \\
0 & w_{0} & 0 & -\lambda+2 g_{2}-\mu_{0} \\
-\lambda+6 g_{1}-\mu_{0} & 0 & -w_{0} & 0 \\
0 & -\lambda+6 g_{2}-\mu_{0} & 0 & -w_{0}
\end{array}\right) \vec{b}=\overrightarrow{0}
$$

where $\vec{b}=\left(\begin{array}{llll}b_{1} & b_{2} & b_{3} & b_{4}\end{array}\right)^{T}$ and $\overrightarrow{0}$ is the zero vector. Using the half-angle trigonometric identities, $g_{1}$ and $g_{2}$ can be rewritten as

$$
\begin{aligned}
& g_{1}=I_{1}-I_{2}=\frac{1}{L} \int_{0}^{L} \phi^{2}(x) d x-\frac{1}{L} \int_{0}^{L} \phi^{2}(x) \cos (2 \mu x) d x, \\
& g_{2}=I_{1}+I_{2}=\frac{1}{L} \int_{0}^{L} \phi^{2}(x) d x+\frac{1}{L} \int_{0}^{L} \phi^{2}(x) \cos (2 \mu x) d x,
\end{aligned}
$$

where $L$ represents the period of the unperturbed solution $\phi$.

Analytic expressions for $I_{2}$ corresponding to the solutions given in (4)-(6) are not known, but the Riemann-Lebesgue Lemma [9] can be used to establish that $I_{2} \sim 0$ for large $\mu$ (large $\rho$ ). This establishes that $g_{1} \sim I_{1}$ and $g_{2} \sim I_{1}$ in the large- $\rho$ limit. For the solution given in (4),

$$
I_{1}=\alpha \frac{4 E(k)+4\left(k^{2}-1\right) K(k)}{4 K(k)},
$$

for the solution given in (5),

$$
I_{1}=\alpha \frac{E(k)}{K(k)}
$$


and for the solution given in (6),

$$
I_{1}=|\alpha| \frac{K(k)-E(k)}{K(k)} .
$$

In each of these expressions, $E(k)$ is the complete elliptic integral of the second kind [3] defined by

$$
E(k)=\int_{0}^{\pi / 2} \sqrt{1-k^{2} \sin ^{2}(\theta)} d \theta .
$$

In order for a nonzero solution of (30) to exist, $w_{0}$ must be chosen to satisfy

$$
w_{0} \sim \pm \sqrt{2 I_{1}-\lambda+\mu_{0}} \sqrt{\lambda-6 I_{1}-\mu_{0}}
$$

where we have used the relationships $g_{1} \sim I_{1}$ and $g_{2} \sim I_{1}$. Maximizing the positive root of $(36)$ with respect to the free parameter $\mu_{0}$ gives

$$
w_{0_{m}} \sim 2 I_{1}
$$

when $\mu_{0} \sim \lambda-4 I_{1}$. Plots of the scaled growth rate $w_{0_{m}} /|\alpha|$ versus $k$ for the solutions given in (4)-(6) are included in Figure 2.

Then $(26 \mathrm{~d})$ defines $\rho_{\eta}$, the value of $\rho$ at which the unstable mode with wave number $\eta$ achieves its maximal growth rate

$$
-\beta \rho_{\eta}^{2} \sim \alpha(2 \pi \eta / L)^{2}+4 I_{1}-\lambda
$$

Equation (38) is valid for all values of $\eta$ such that $\rho$ is large. If $\eta$ is an integer, then the corresponding perturbation has the same period as $\phi$. If $\eta$ is not an integer, then the perturbation has an $x$-period different from $\phi$. We also find how far $\rho$ can deviate from $\rho_{\eta}$ before $\omega_{0}$ becomes imaginary

$$
\delta \rho_{\eta} \sim I_{1} /\left(|\beta| \rho_{\eta}\right)=O(1 / \eta)
$$

\section{Remarks:}

- If $\alpha \beta>0$, then all one-dimensional trivial-phase solutions are unstable with respect to two-dimensional perturbations only if the transverse wavelength is longer than a well-defined cutoff.

- This asymptotic argument establishes that all one-dimensional trivial-phase solutions with $k<1$ are unstable with respect to two-dimensional perturbations with arbitrarily short wavelengths if $\alpha \beta<0$. 


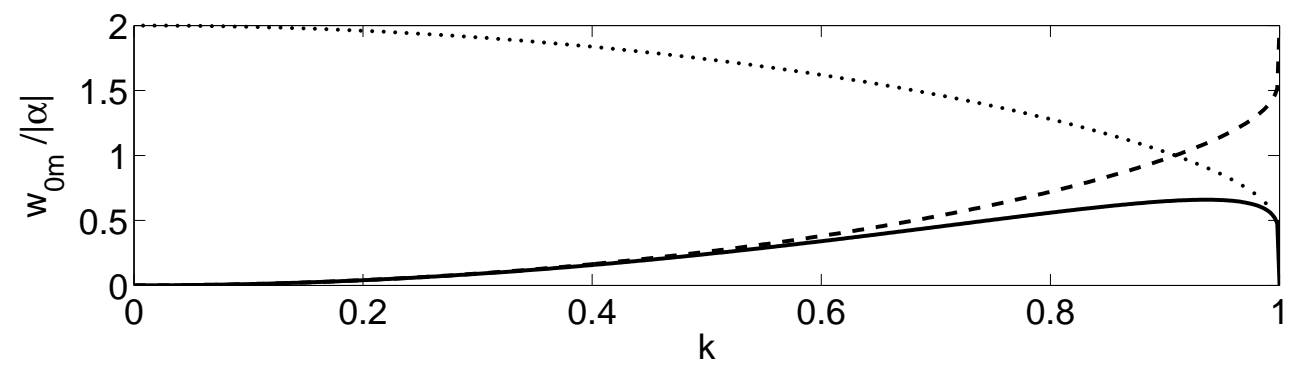

Fig. 2. Plots of $w_{0_{m}} /|\alpha|$ versus $k$. The solid line corresponds to (4), the dotted line corresponds to (5) and the dashed line corresponds to (6).

- In the large- $\rho$ limit, the perturbations to which the solutions given in (4)-(6) are unstable have short wavelengths in both the transverse and longitudinal directions.

- For all values of $k$, the scaled growth rates of the instabilities remain bounded as $\rho \rightarrow \infty$. Specifically, $0 \leq w_{0_{m}} \leq 2|\alpha|$ for all solutions under consideration.

- The $k \rightarrow 1$ limit of the large- $\rho$ results is singular because the period of the unperturbed solution goes to infinity and "large $\rho$ " loses its meaning.

\section{Numerical results}

In order to find approximations to the spectra of (11) corresponding to the solutions given in (4)-(6), we employ the numerical method of Deconinck \& Kutz [6]. In Section 4.1, we describe this method in detail. In Sections 4.2 and 4.3 , we include results of numerical studies of (11) corresponding to the solutions given in (4)-(6).

\subsection{Numerical method}

The Fourier-Floquet-Hill (FFH) numerical method presented by Deconinck \& Kutz [6] relies on Fourier and Floquet theory. We chose this method because it is spectrally accurate for differential eigenvalue problems with periodic coefficients and is a significant improvement over the method of Carter \& Segur [4].

The only nonconstant coefficient in $(11)$ is $\phi^{2}$. The Fourier series for $\phi^{2}$ corresponding to the solutions given in (4)-(6) can be obtained from the relationships

$$
\begin{gathered}
\operatorname{sn}^{2}(x, k)=\frac{1-E(k) / K(k)}{k^{2}}-\frac{2 \pi^{2}}{k^{2} K^{2}(k)} \sum_{m=1}^{\infty} \frac{m q^{m}}{1-q^{2 m}} \cos \left(\frac{m \pi x}{K(k)}\right), \\
\operatorname{cn}^{2}(x, k)=1-\operatorname{sn}^{2}(x, k),
\end{gathered}
$$




$$
\operatorname{dn}^{2}(x, k)=1-k^{2} \operatorname{sn}^{2}(x, k)
$$

where

$$
q=\exp \left(-\frac{\pi K\left(\sqrt{1-k^{2}}\right)}{K(k)}\right)
$$

Equation (40a) is found in [11] and it should be noted that [3] contains an incorrect expression (Equation 911.01) for the same Fourier series. Floquet's Theorem establishes that all bounded solutions of (11) are of the form

$$
\begin{aligned}
& U(x)=\mathrm{e}^{i \mu x} \sum_{j=-\infty}^{\infty} \hat{U}_{j} \mathrm{e}^{i \pi j x / L}, \\
& V(x)=\mathrm{e}^{i \mu x} \sum_{j=-\infty}^{\infty} \hat{V}_{j} \mathrm{e}^{i \pi j x / L},
\end{aligned}
$$

where $\mu \in[-\pi / L, \pi / L)$. We refer to functions that share the period $L$ of $\phi^{2}(x)$ as periodic; functions that are periodic with period $2 L$ are called antiperiodic. It follows that if $\mu=0$, then $U$ and $V$ are either periodic or antiperiodic functions of $x$. For all other values of $\mu$, the functions $U$ and $V$ are quasiperiodic or have periods exceeding $2 L$.

Substituting (42) and the appropriate series for $\phi^{2}$ into (11) and multiplying the resulting equations through by $\mathrm{e}^{-i \mu x}$ leads to the following system of coupled difference equations

$$
\begin{gathered}
\left(\lambda+\beta \rho^{2}+\alpha\left(\mu+\frac{\pi j}{L}\right)^{2}\right) \hat{V}_{j}-2 \sum_{m=-\infty}^{\infty} \hat{f}_{(j-m) / 2} \delta_{2 \mid(j-m)} \hat{V}_{m}=\Omega \hat{U}_{j}, \\
-\left(\lambda+\beta \rho^{2}+\alpha\left(\mu+\frac{\pi j}{L}\right)^{2}\right) \hat{U}_{j}+6 \sum_{m=-\infty}^{\infty} \hat{f}_{(j-m) / 2} \delta_{2 \mid(j-m)} \hat{U}_{m}=\Omega \hat{V}_{j},
\end{gathered}
$$

where $j$ is any integer, $\hat{f}_{l}$ is the $l^{\text {th }}$ - Fourier coefficient of $\phi^{2}$, and $\delta_{2 \mid(j-m)}$ is 1 if 2 divides $(j-m)$ and 0 otherwise. The system given in (43) is a doubly bi-infinite set of difference equations that is equivalent to (11).

For numerical purposes, we restrict $j \in[-N, N]$ where $N$ is a large positive integer. For any given unperturbed solution (i.e. any $\alpha, \beta$ and $k$ ), this reduces (43) to a $(4 N+2)$-dimensional linear system with two parameters, $\rho$ and $\mu$. The $\rho$-interval (We choose $\rho \in[0,2]$ if $\alpha \beta>0$ and $\rho \in[0,4]$ if $\alpha \beta<0$.) is divided into $N_{\rho}$ equally spaced gridpoints. For each $\rho$, the $\mu$-interval $[-\pi / L, \pi / L)$ is divided into $N_{\mu}$ equally spaced gridpoints. The eigenvalues (all $4 N+2$ of them) of the coefficient matrix in the truncated version of (43) are found for each gridpoint in $(\rho, \mu)$-space. For each choice of $\alpha, \beta$ and unperturbed solution, $N_{\rho} \cdot N_{\mu}$ matrix eigenvalue problems are solved resulting in a total of $N_{\rho} \cdot N_{\mu} \cdot(4 N+2)$ eigenvalues.

The spectrum of a one-dimensional linear operator (or more correctly, its maximal extension [14]) consists of a collection of straight-line segments (usually 
confined to the coordinate axes) and curves. We define a spectral component to be a subset of the spectrum that is connected to a spectral element corresponding to a periodic eigenfunction, without passing through a spectral element corresponding to an antiperiodic eigenfunction. Note that there are an infinite number of spectral components. The consequence of the truncation of $(43)$ to a $(4 N+2)$-dimensional system is to compute approximations to only $4 N+2$ spectral components. This truncation effect is the same as that of using finite difference methods [6]. Varying $\mu$ results in approximate spectral elements on the same collection of spectral components.

Figure 3a contains a plot of $\Re(\Omega)>0$ versus $\rho$. Many figures like this are shown in what follows and it is essential that the reader has a clear understanding of what is displayed. For any given choice of $\alpha$ and $\beta$ (here $\alpha=-\beta=-1$ ), an approximation to the spectrum of (11) is computed. This is achieved using the FFH method as described above. For Fig. 3a, $N_{\rho}=800$ and $N_{\mu}=20$. As we are interested in instabilities of the solutions of (1), the real part of any element of the spectrum found is of most interest to us. To present this information compactly, the spectrum is projected onto the real axis, and values with positive projections are kept. Thus for any chosen value of $\rho$ a point set (due to the numerical approximation) of positive values is obtained. For each fixed value of $\rho$, these point sets are plotted vertically, see Fig. 3a. In this figure, the gray and black points correspond to the real projections of real and (non-real) complex values respectively.

Traditionally, special attention is devoted to periodic or antiperiodic eigenfunctions. As remarked earlier, these are easily obtained using the FFH method, by letting $\mu=0$ in (42). Using the same construction as above with $N_{\mu}=$ $1, \mu=0$ results in Fig. 3b. Note that no projections of non-real complex were included in Fig. 3b. By virtue of this more restrictive construction, all point sets displayed in Fig. 3b are subsets of those in Fig. 3a. The comparison of the two figures illustrates the importance of considering eigenfunctions beyond those that are periodic or antiperiodic. Alternatively, Fig. 3b can be obtained using the monodromy method used by Carter \& Segur [4] and described in more detail in [12].

To emphasize the efficiency of the FFH method, we note that even though the data displayed in Fig. 3a obtained by the FFH method contains nearly 20 times as much information, it is significantly faster to compute than the data shown in Fig. 3b using the monodromy method. Computing the data for Figure 3a using the FFH method requires approximately 120 seconds on a 3.2 GHz Pentium 4 with 1.0GB RAM running Mathematica 5.0 on Microsoft Windows XP Professional. The numerical parameters used in simulations to create Fig. 3a are included in Appendix B. 
(a)

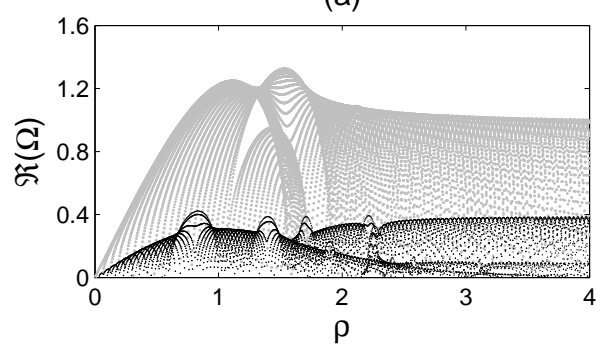

(b)

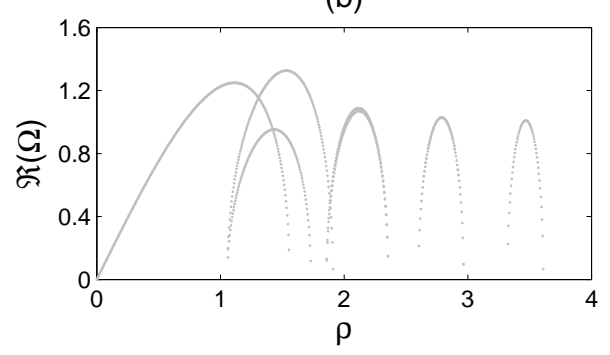

Fig. 3. Plots of $\Re(\Omega)>0$ versus $\rho$ obtained using the Fourier-Floquet-Hill method (a) and the monodromy method (b).

\subsection{Case I: $\boldsymbol{\alpha} \boldsymbol{\beta}>\mathbf{0}$}

\section{Solutions of cn-type}

This section contains results from numerical simulations using $\alpha=\beta=1$ and solutions of the form given in (4). Figure 4 contains plots of $\Re(\Omega)>0$ versus $\rho$ for $k=0.2,0.5,0.9$ and 0.9999 . The gray points correspond to real values of $\Omega$ and the black points correspond to non-real values of $\Omega$. The solid lines are obtained from the small- $\rho$ asymptotic results. A record of all numerical parameters is included in Appendix B.

\section{Observations:}

- For all values of $k$ there is a critical value of $\rho=\rho_{c}$, above which there is no apparent instability.

- As $k$ increases, $\rho_{c}$ increases to approximately 1.75 .

- As $k$ increases, the maximum growth rate over all $\rho$ increases to approximately 1.54 .

- As $k$ increases, the value of $\rho$ at which the maximum growth rate is achieved increases to approximately 1.21.

- For small values of $\rho$, complex eigenvalues are dominant (i.e. have the largest growth rates).

- The unperturbed solution is unstable with respect to one-dimensional ( $\rho=$ 0) perturbations. This instability is an oscillatory instability.

- As $k \rightarrow 1$, the growth-rate regions of the spectrum limit to a single realvalued curve.

- As $k \rightarrow 1$, the spectrum for the two-dimensional problem (i.e. including all values of $\rho$ ) appears to approach $[-\pi / 2, \pi / 2]$ on the real line. For the one-dimensional problem (fixed $\rho$ ) there are only two isolated eigenvalues on the real line.

- As $k \rightarrow 1$, the spectra for the solutions given in (4) and (5) limit to the same spectrum.

- There is strong agreement between the numerical and small- $\rho$ asymptotic 

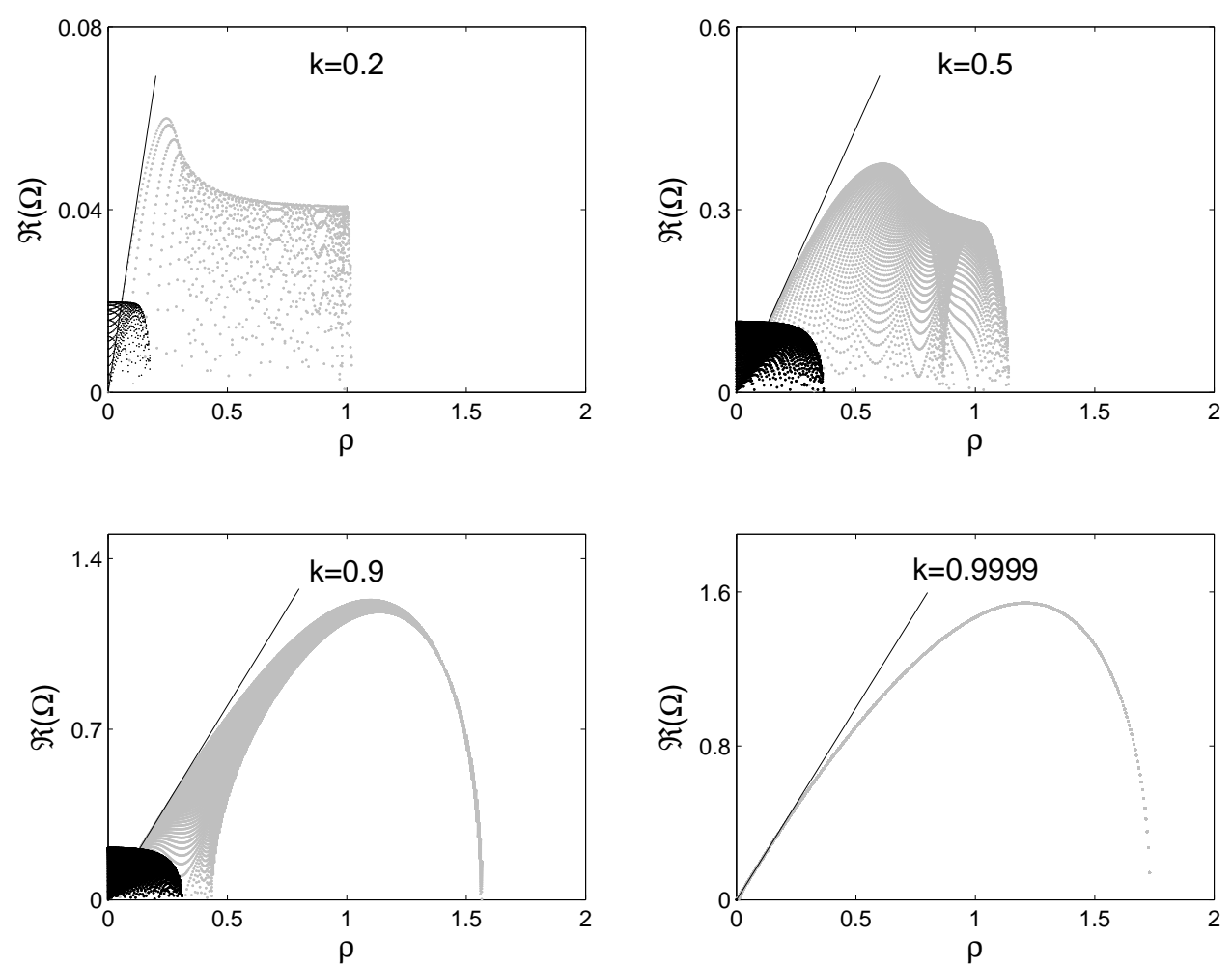

Fig. 4. Plots of $\Re(\Omega)>0$ versus $\rho$ for four different values of $k$ corresponding to solutions of cn-type with $\alpha=\beta=1$. The spectra were computed for $\rho \in[0,2]$. The sharp drop off of $\Re(\Omega)$ is genuine.

results.

- In the small- $\rho$ limit, the asymptotic results do not select the dominant instability. However, they do select the dominant instability corresponding to real eigenvalues.

\section{Solutions of dn-type}

This section contains results from numerical simulations using $\alpha=\beta=1$ and solutions of the form given in (5). Figure 5 contains plots of $\Re(\Omega)>0$ versus $\rho$ for $k=0.2,0.5,0.9$ and 0.9999 . The gray points correspond to real values of $\Omega$ and the black points correspond to non-real values of $\Omega$ with nonzero imaginary part. The solid lines are obtained from the small- $\rho$ asymptotic results. A record of all numerical parameters is included in Appendix B.

\section{Observations:}

- For all values of $k$, there is a critical value of $\rho=\rho_{c}$, above which there is no apparent instability. 
- As $k$ increases, the maximum growth rate over all $\rho$ decreases to approximately 1.54 .

- There are no oscillatory instabilities.

- The unperturbed solution is unstable with respect to one-dimensional ( $\rho=$ 0) perturbations.

- As $k \rightarrow 0$, the spectrum approaches the (known) spectrum for the Stokes' wave solution. For the Stokes' wave of amplitude 1, the unstable modes fill the region above the $\rho$-axis and below the curve given by (for $0 \leq \rho \leq \sqrt{2}$ ) $\Re(\Omega)=\rho \sqrt{4-\rho^{2}}$ and (for $\left.\rho>\sqrt{2}\right) \Re(\Omega)=2[5]$.

- As $k \rightarrow 1$, the growth-rate regions of the spectrum limit to a single realvalued curve.

- As $k \rightarrow 1$, the spectrum for the two-dimensional problem (i.e. including all values of $\rho$ ) appears to approach $[-\pi / 2, \pi / 2]$ on the real line. For the one-dimensional problem (fixed $\rho$ ) there are only two isolated eigenvalues on the real line.

- As $k \rightarrow 1$, the spectra for the solutions given in (4) and (5) limit to the same spectrum.

- Comparing the small- $\rho$ asymptotic results with the numerical results is difficult because the asymptotic results are in the middle of continua.

- In the small- $\rho$ limit, the asymptotic results do not select the dominant instability.

\section{Solutions of sn-type}

This section contains results from numerical simulations using $\alpha=\beta=-1$ and solutions of the form given in (6). Figure 6 contains plots of $\Re(\Omega)>0$ versus $\rho$ for $k=0.2,0.5,0.9$ and 0.9999 . The gray points correspond to real values of $\Omega$ and the black points correspond to non-real values of $\Omega$ with nonzero imaginary part. The solid lines are obtained from the small- $\rho$ asymptotic results. A record of all numerical parameters is included in Appendix B.

\section{Observations:}

- For all values of $k$, there is a critical value of $\rho=\rho_{c}$, above which there is no apparent instability.

- For all values of $k$, it appears that $\rho_{c}=1$.

- As $k$ increases, the maximum growth rate over all $\rho$ increases to approximately 0.52 .

- As $k$ increases, the value of $\rho$ at which the maximum growth rate is achieved decreases to approximately 0.7 .

- The unperturbed solution is spectrally stable with respect to one-dimensional $(\rho=0)$ perturbations.

- There are no oscillatory instabilities. 

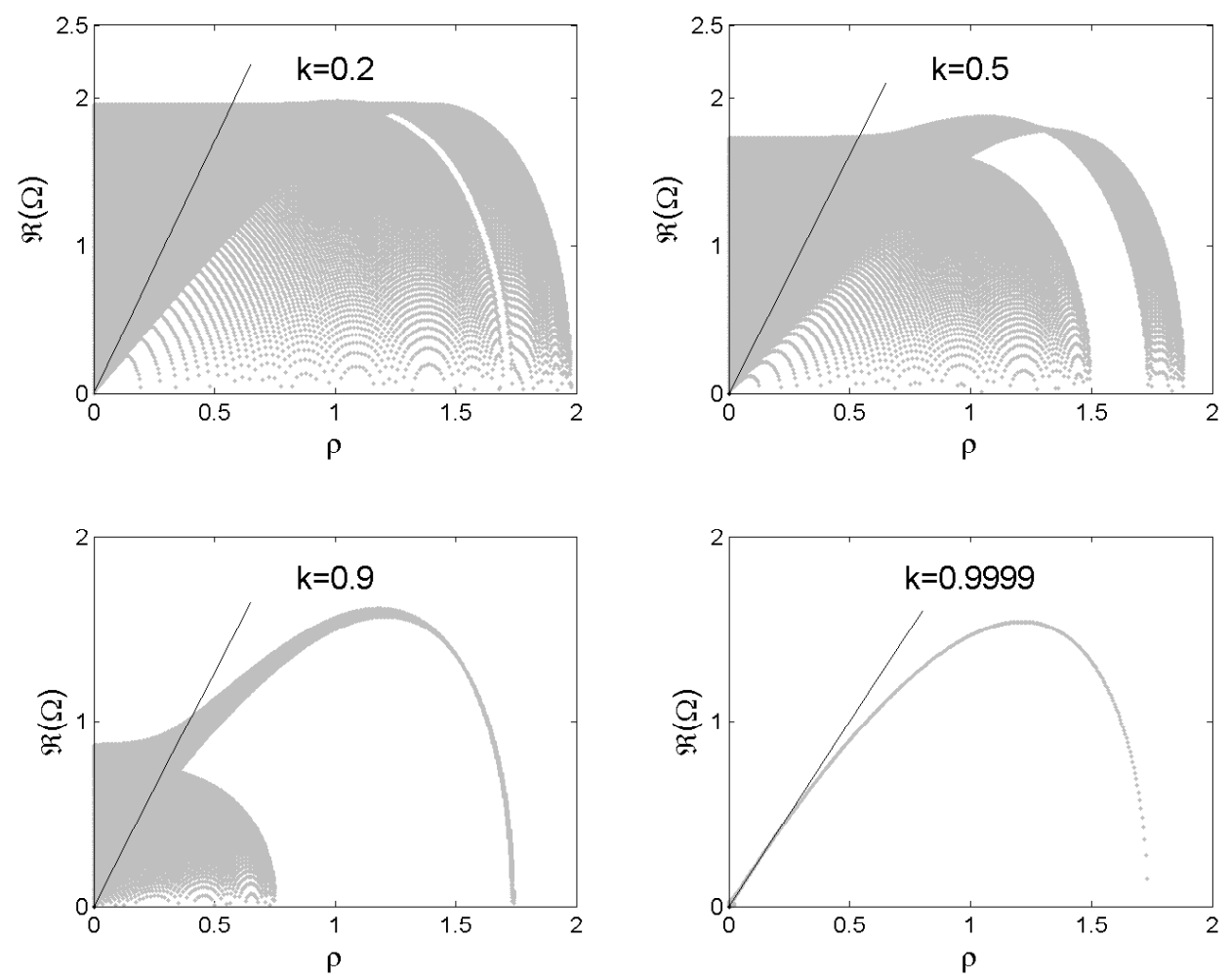

Fig. 5. Plots of $\Re(\Omega)>0$ versus $\rho$ for four different values of $k$ corresponding to solutions of dn-type with $\alpha=\beta=1$.

- As $k \rightarrow 1$, the growth-rate regions of the spectrum limit to a single realvalued curve.

- There is strong agreement between the numerical and small- $\rho$ asymptotic results.

- In the small- $\rho$ limit, the asymptotic results select the dominant instability.

\subsection{Case II: $\boldsymbol{\alpha} \boldsymbol{\beta}<\mathbf{0}$}

\section{Solutions of cn-type}

This section contains results from numerical simulations using $\alpha=-\beta=1$ and solutions of the form given in (4). The $k=1$ version of this case is studied in detail in [7]. Figure 7 contains plots of $\Re(\Omega)>0$ versus $\rho$ for $k=0.2,0.5,0.9$ and 0.9999 . The gray points correspond to real values of $\Omega$ and the black points correspond to non-real values of $\Omega$. The solid lines are obtained from the small- $\rho$ asymptotic results and the dashed lines are obtained using the large- $\rho$ asymptotic results. A record of all numerical parameters is included in 

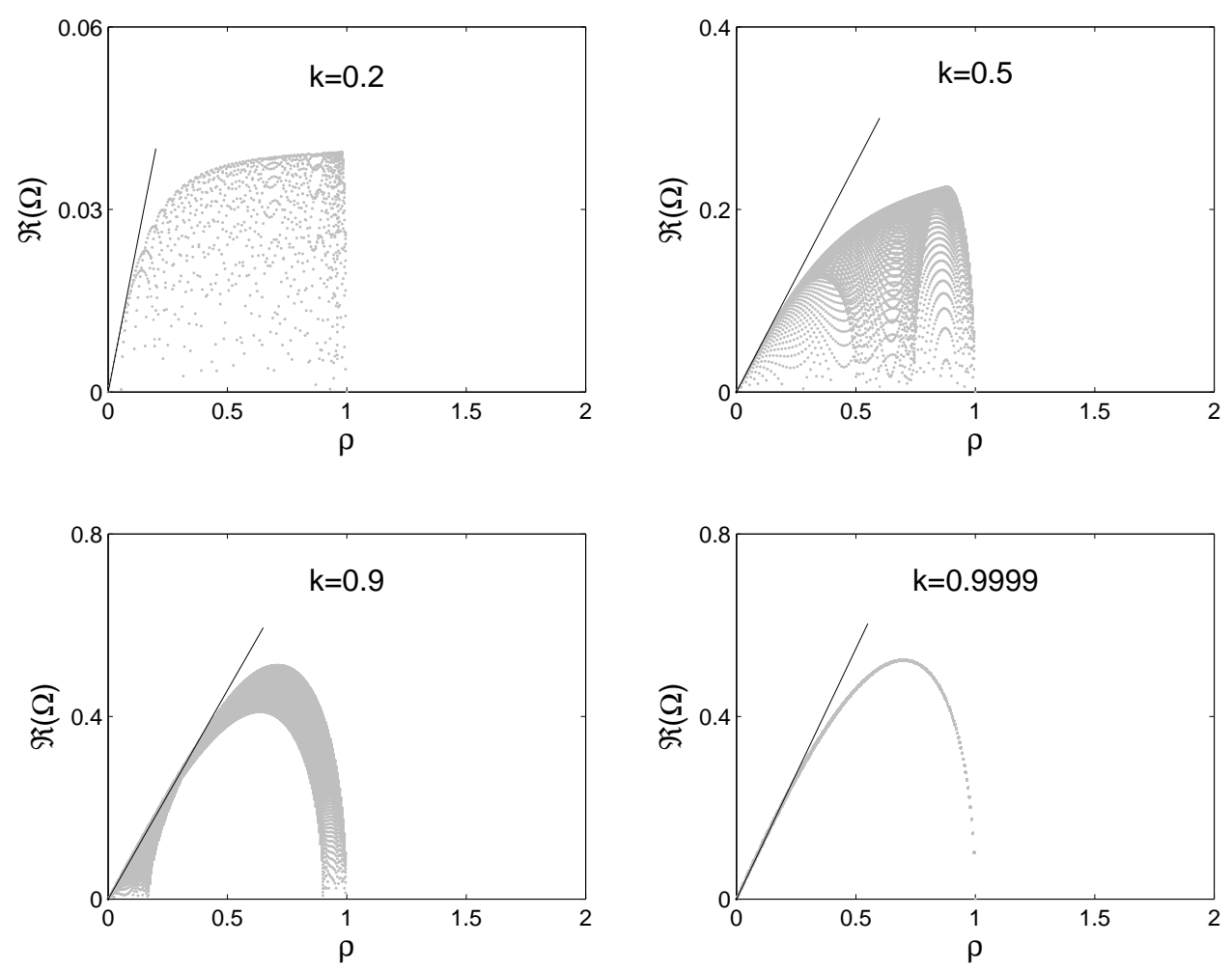

Fig. 6. Plots of $\Re(\Omega)>0$ versus $\rho$ for four different values of $k$ corresponding to solutions of sn-type with $\alpha=\beta=-1$. The spectra were computed for $\rho \in[0,2]$. The sharp drop off of $\Re(\Omega)$ is genuine.

Appendix B.

\section{Observations:}

- For all values of $k$, there does not appear to be a $\rho_{c}$ above which there are no instabilities.

- For all values of $k$, as $\rho$ increases, $\Re(\Omega)$ approaches a finite nonzero limit.

- As $k$ increases, the maximum growth rate over all $\rho$ decreases to approximately 0.66 .

- Oscillatory instabilities exist for all values of $k$.

- For small values of $\rho$, oscillatory instabilities dominate other instabilities.

- The unperturbed solution is unstable with respect to one-dimensional ( $\rho=$ 0) perturbations. This instability is an oscillatory instability.

- As $k \rightarrow 1$, the spectra for the solutions given in (4) and (5) limit to the same spectrum.

- There is strong agreement between the numerical and small- $\rho$ asymptotic results.

- In the small- $\rho$ limit, the asymptotic results do not select the dominant 

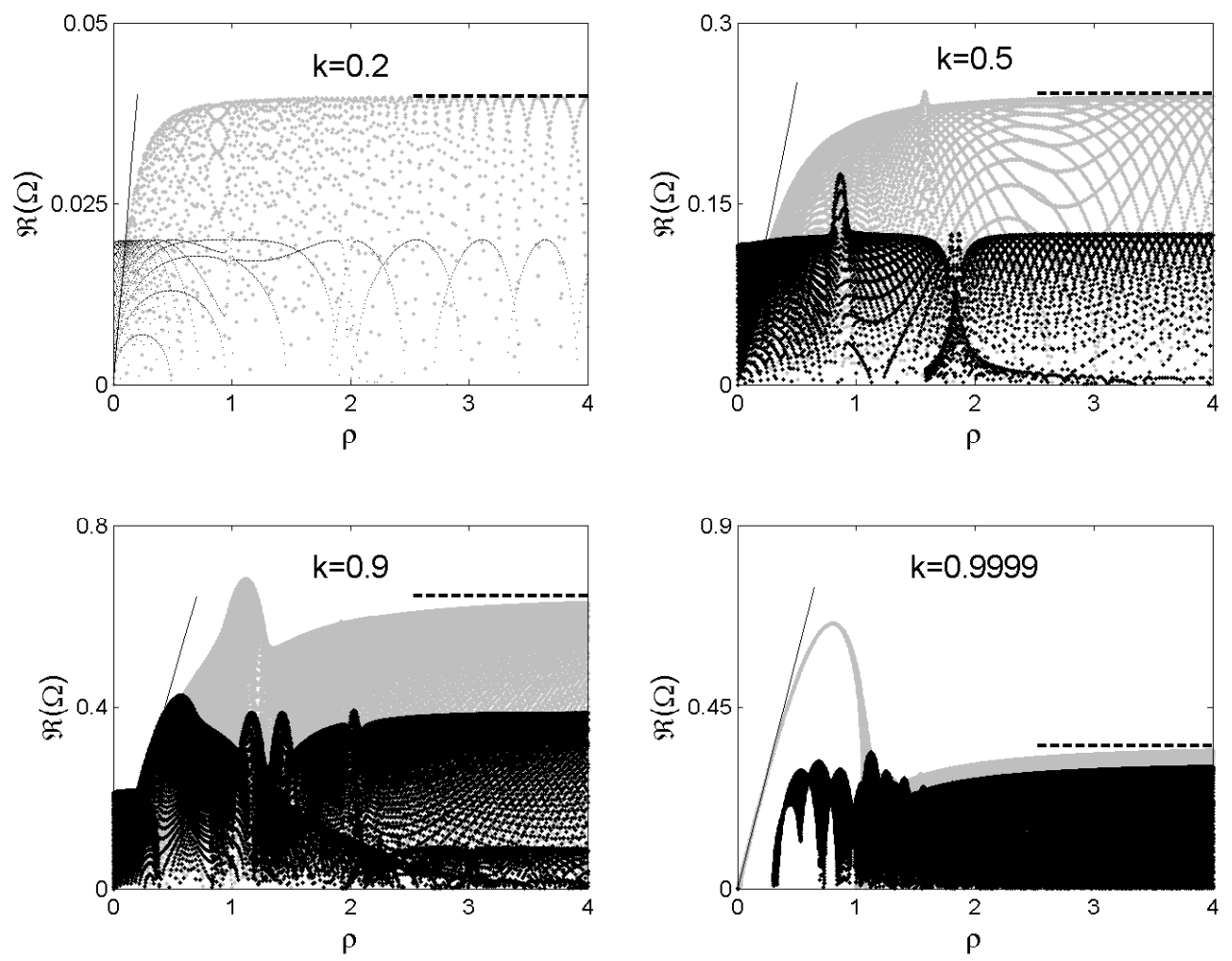

Fig. 7. Plots of $\Re(\Omega)>0$ versus $\rho$ for four different values of $k$ corresponding to solutions of cn-type with $\alpha=-\beta=1$.

instability. However, they do select the dominant instability corresponding to real eigenvalues.

- There is strong agreement between the numerical and large- $\rho$ asymptotic results.

\section{Solutions of dn-type}

This section contains results from numerical simulations using $\alpha=-\beta=1$ and solutions of the form given in (5). The $k=1$ version of this solution is studied in detail in [7]. Figure 8 contains plots of $\Re(\Omega)>0$ versus $\rho$ for $k=0.2,0.5,0.9$ and 0.9999 . The gray points correspond to real values of $\Omega$ and the black points correspond to non-real values of $\Omega$. The solid lines are obtained from the small- $\rho$ asymptotic results and the dashed lines are obtained using the large- $\rho$ asymptotic results. A record of all numerical parameters is included in Appendix B.

\section{Observations:}

- For all values of $k$, there does not appear to be a $\rho_{c}$ above which there are 

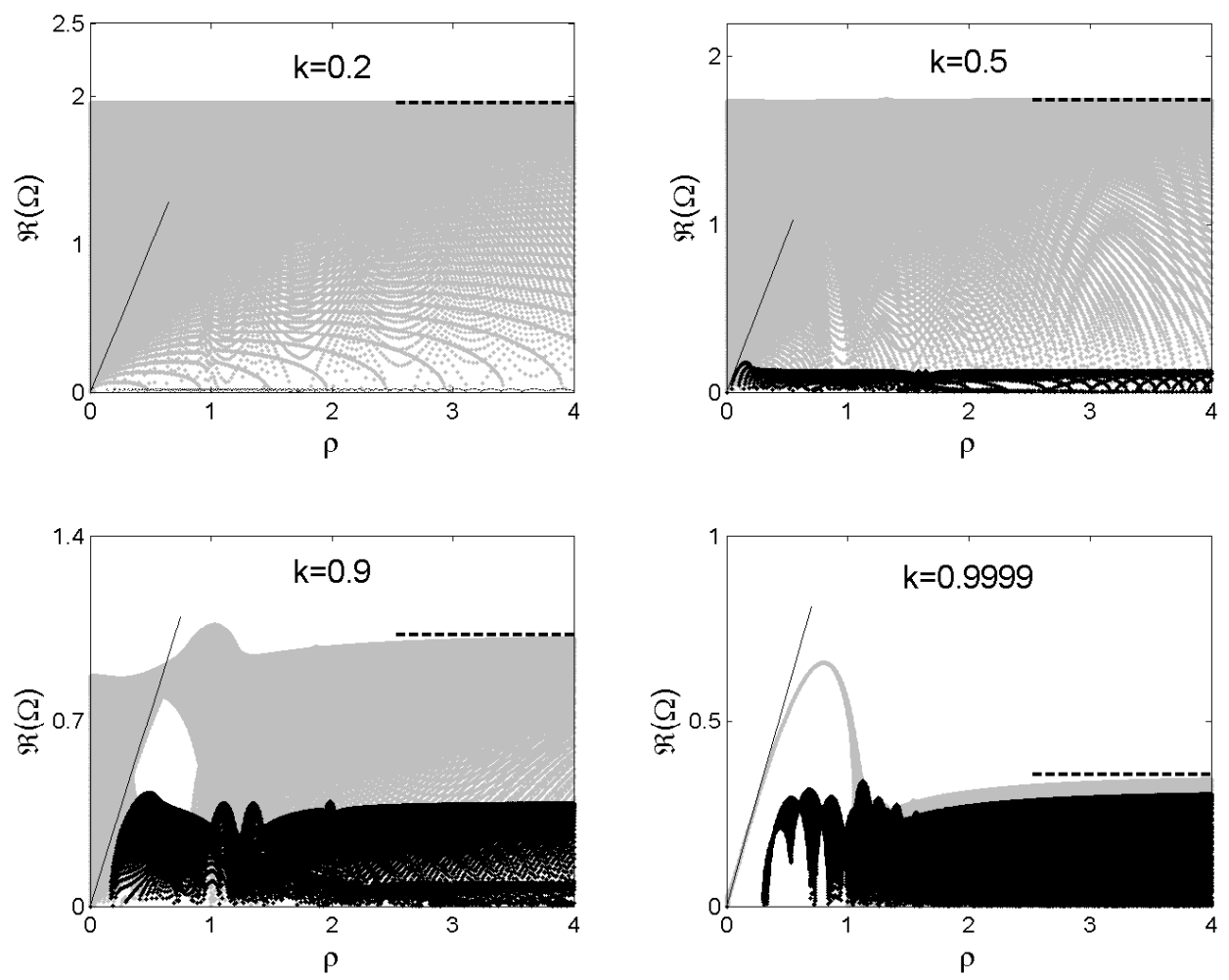

Fig. 8. Plots of $\Re(\Omega)>0$ versus $\rho$ for four different values of $k$ corresponding to solutions of dn-type with $\alpha=-\beta=1$.

no instabilities.

- As $k \rightarrow 0$, the spectrum approaches the (known) spectrum for the Stokes' wave solution. For the Stokes' wave of amplitude 1, the unstable modes fill the region above the $\rho$-axis and below the curve given by (for $0 \leq \rho \leq \sqrt{2}$ ) $\Re(\Omega)=\rho \sqrt{4-\rho^{2}}$ and (for $\left.\rho>\sqrt{2}\right) \Re(\Omega)=2[5]$.

- The unperturbed solution is unstable with respect to one-dimensional ( $\rho=$ 0) perturbations.

- For all values of $k$, as $\rho$ increases, $\Re(\Omega)$ approaches a finite nonzero limit.

- As $k \rightarrow 1$ the maximal growth rate over all $\rho$ decreases to approximately 0.66 .

- Oscillatory instabilities exist for all values of $k$.

- As $k \rightarrow 1$, the spectra for the solutions given in (4) and (5) limit to the same spectrum.

- Comparing the small- $\rho$ asymptotic results with the numerical results is difficult because the asymptotic result is in the middle of a continuum.

- In the small- $\rho$ limit, the asymptotic results do not select the dominant instability.

- There is strong agreement between the numerical and large- $\rho$ asymptotic results. 


\section{Solutions of sn-type}

This section contains results from numerical simulations using $\alpha=-\beta=-1$ and solutions of the form given in (6). Figure 9 contains plots of $\Re(\Omega)>0$ versus $\rho$ for $k=0.2,0.5,0.9$ and 0.9999 . The gray points correspond to real values of $\Omega$ and the black points correspond to non-real values of $\Omega$. The solid lines are obtained from the small- $\rho$ asymptotic results and the dashed lines are obtained using the large- $\rho$ asymptotic results. A record of all numerical parameters is included in Appendix B.

\section{Observations:}

- For all values of $k$, there does not appear to be a $\rho_{c}$ above which there are no instabilities.

- As $k$ increases the maximum growth rate over all $\rho$ increases limiting to approximately 2.

- Oscillatory instabilities exist for all values of $k$, but they never dominate.

- The unperturbed solution is spectrally stable with respect to one-dimensional $(\rho=0)$ perturbations.

- As $k \rightarrow 1$, the spectrum approaches the (known) spectrum for the Stokes' wave solution. For the Stokes' wave of amplitude 1, the unstable modes fill the region above the $\rho$-axis and below the curve given by (for $0 \leq \rho \leq \sqrt{2}$ ) $\Re(\Omega)=\rho \sqrt{4-\rho^{2}}$ and (for $\left.\rho>\sqrt{2}\right) \Re(\Omega)=2[5]$.

- For all values of $k$, as $\rho$ increases, $\Re(\Omega)$ approaches a finite nonzero limit.

- There is strong agreement between the numerical and small- $\rho$ asymptotic results.

- In the small- $\rho$ limit, the asymptotic results select the dominant instability.

- There is strong agreement between the numerical and large- $\rho$ asymptotic results.

\section{A conjecture about the topology of the spectrum}

Based on the multitude of runs we investigated, only some of which are presented here, we can formulate a conjecture about the topology of the spectra of (11). To do so, we need to introduce some terminology.

It is known that the spectrum of a second-order self-adjoint linear differential operator with periodic coefficients consists of a sequence of bands confined to a real half-line [17]. It is also known that the edges of these bands are eigenvalues corresponding to either periodic or antiperiodic eigenfunctions. Thus, in that context, special importance is associated with these periodic or antiperiodic eigenfunctions. To what extent can the same be said about our 

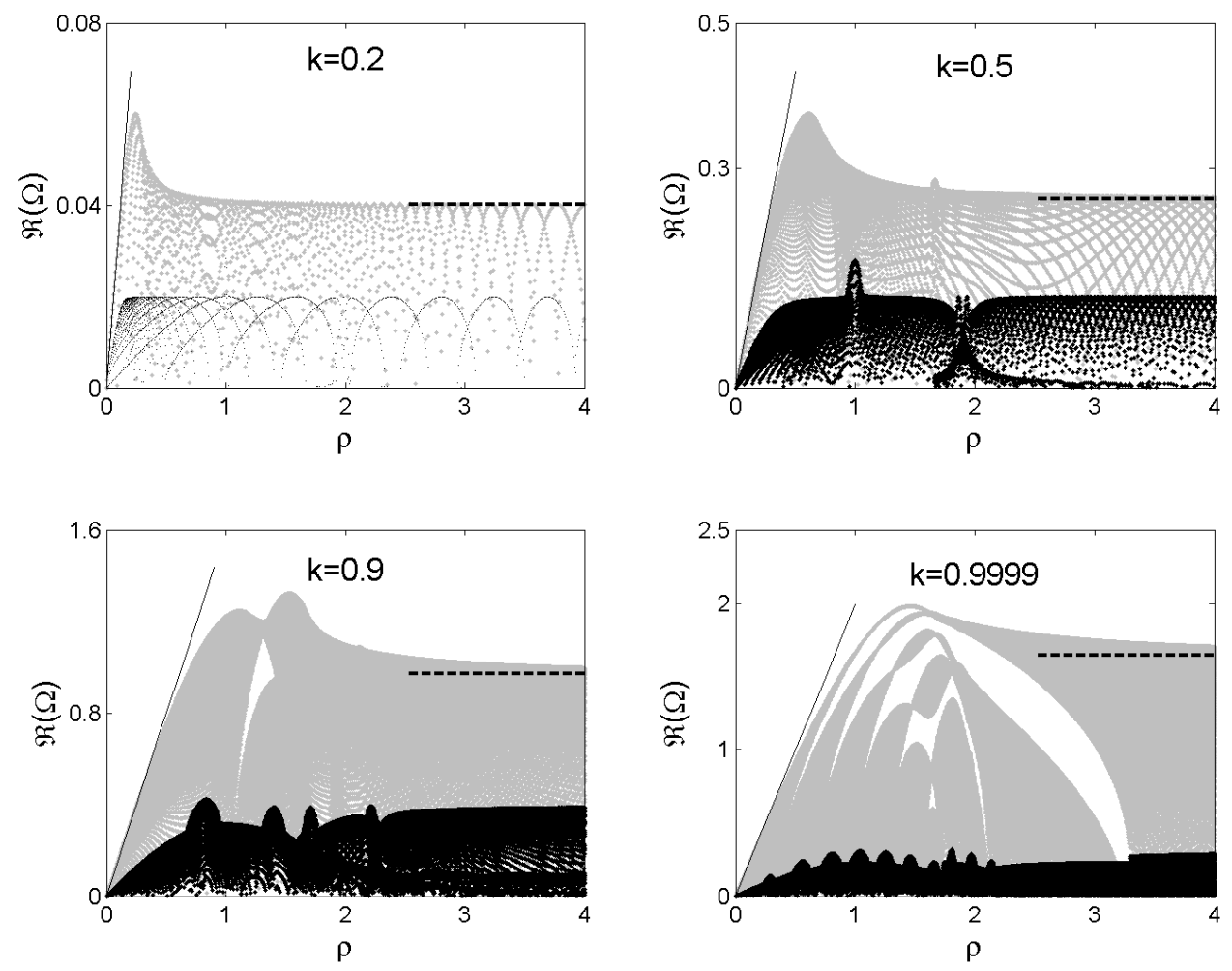

Fig. 9. Plots of $\Re(\Omega)>0$ versus $\rho$ for four different values of $k$ corresponding to solutions of sn-type with $\alpha=-\beta=-1$.

spectral problem, which is of fourth order?

Definition. (Periodic or antiperiodic curves) Periodic or antiperiodic curves are curves in the $(\rho, \Re(\Omega))$-plane consisting of points $(\rho, \Omega)$ with $\Omega \in \mathbb{R}$, so that $\Omega$ is a spectral element corresponding to a periodic or antiperiodic eigenfunction.

For instance, to the eye Fig. 3b displays at least seven periodic or antiperiodic curves, two of which are almost identical.

Definition. (Zakharov-Rubenchik curve) The Zakharov-Rubenchik curve is the periodic or antiperiodic curve that passes through the origin of the $(\rho, \Re(\Omega))$-plane.

Note that in all cases, we have an asymptotic approximation to the ZakharovRubenchik curve, given by (23c) or (24c). The question we want to address is how important the knowledge of these curves is in terms of understanding the entire collection of unstable real modes, as shown in gray in Fig. 3a. 
We claim the following conjecture holds:

Conjecture: (i) Any area in the $\left(\rho, \Omega \in \mathbb{R}^{+}\right)$-plane which is contained under an odd number of periodic or antiperiodic curves, one of which is the Zakharov-Rubenchik curve, is filled with unstable modes. (ii) The boundary of any bounded areas containing spectrally stable modes consists of periodic or antiperiodic curves and possibly the horizontal axis.

Note that it is not true that any area in the $\left(\rho, \Omega \in \mathbb{R}^{+}\right)$-plane which is contained under an even number of periodic or antiperiodic curves, one of which is the Zakharov-Rubenchik curve, is filled with stable modes.

This conjecture, if correct, may be used to infer information about unstable modes that are not periodic or antiperiodic from information about periodic or antiperiodic instabilities.

\section{Summary}

In this paper, we

- Established asymptotically that all periodic one-dimensional trivial-phase solutions of NLS are unstable with respect to two-dimensional perturbations with long wavelengths in the transverse dimension regardless of the signs of $\alpha$ and $\beta$. If $\beta>0$, then the solution is unstable with respect to a growing "neck" mode. If $\beta<0$, then the solution is unstable with respect to a growing "snake" mode.

- Established asymptotically that if $\alpha \beta<0$, then all periodic one-dimensional trivial-phase solutions of NLS are unstable with respect to two-dimensional perturbations with arbitrarily short wavelengths. Further, as the wavelength of the perturbation decreases, the growth rate of the instability approaches a finite nonzero value.

- Determined numerically the linear stability spectrum for twelve periodic trivial-phase solutions. We present results for periodic and nonperiodic perturbations. To our knowledge, these are the most complete and detailed spectra available. Our observations are based upon many numerical simulations which are not explicitly shown here. All spectra resulting from these numerical simulations are well-represented by those included.

- Established numerically that the solution given in (4) is unstable with respect to one-dimensional perturbations. This instability is oscillatory in nature because its growth rate is complex.

- Established numerically that the solution given in (5) is unstable with respect to one-dimensional perturbations. 
- Established numerically that the solution given in (6) is spectrally stable with respect to one-dimensional perturbations.

- Established numerically that the solution given in (4) is unstable with respect to oscillatory instabilities regardless of the sign of $\beta$.

- Established numerically that the solutions given in (5) and (6) are unstable with respect to oscillatory instabilities only if $\beta<0$.

\section{Acknowledgments}

This material is based in part upon work supported by the National Science Foundation under grants DMS-0139771 (J.D.C.) and DMS-0351466 (B.D.).

\section{A Definition of the $f_{j}$ 's}

Here we include explicit definitions of all the functions necessary to calculate the small- $\rho$ growth rate. In each of the following expressions, $L$ is the period of the unperturbed solution and $\tilde{\phi}=\phi / \sqrt{|\alpha|}$. Note that the $f_{j}$ 's depend only on the parameter $k$.

$$
\begin{array}{rlrl}
f_{1}(k) & =\frac{1}{L} \int_{0}^{L} \tilde{\phi}^{2} \tilde{\phi}_{x}^{-2} d x, & f_{2}(k)=\frac{1}{L} \int_{0}^{L} \tilde{\phi}_{x}^{-2} d x, \\
f_{3}(k)=\frac{1}{L} \int_{0}^{L} \tilde{\phi}^{-2} d x, & f_{4}(k)=\frac{1}{L} \int_{0}^{L} \tilde{\phi}^{4} \tilde{\phi}_{x}^{-2} d x, \\
f_{5}(k)=\frac{1}{L} \int_{0}^{L} \tilde{\phi}^{2} d x, & f_{6}(k)=\frac{1}{L} \int_{0}^{L} \tilde{\phi}_{x}^{2} d x .
\end{array}
$$

\section{A.1 Solutions of cn-type}

In this section, we specialize the above expressions for the $f_{j}$ to solutions of cn-type (solutions of the form given in (4)). $L=4 K(k), k^{\prime}=\sqrt{1-k^{2}}$ and $\Gamma=E(k) / K(k)$.

$$
\begin{array}{lrl}
f_{1}(k) & =1-2 \Gamma, & f_{2}(k)=\frac{1}{k^{2}{k^{\prime}}^{2}}\left(k^{\prime 2}+\left(k^{2}-k^{\prime 2}\right) \Gamma\right), \\
f_{3}(k)=\frac{1}{k^{2} k^{\prime 2}}\left(k^{\prime 2}-\Gamma\right), & f_{4}(k)=k^{2}\left(1-2 \Gamma-\frac{1}{k^{2}}(1-\Gamma)\right), \\
f_{5}(k)=\Gamma-k^{\prime 2}, & f_{6}(k)=\frac{1}{3}\left(k^{\prime 2}+\left(2 k^{2}-1\right) \Gamma\right) .
\end{array}
$$




\section{A.2 Solutions of dn-type}

In this section, we specialize the above expressions for the $f_{j}$ to solutions of dn-type (solutions of the form given in (5)). $L=2 K(k), k^{\prime}=\sqrt{1-k^{2}}$ and $\Gamma=E(k) / K(k)$.

$f_{1}(k)=\frac{1}{k^{4}}\left(2{k^{\prime}}^{2}-\left(1+{k^{\prime}}^{2}\right) \Gamma+k^{2}(1-\Gamma)\right), \quad f_{2}(k)=\frac{1}{k^{4}{k^{\prime}}^{2}}\left(2{k^{\prime}}^{2}-\left(1+{k^{\prime}}^{2}\right) \Gamma\right)$,

$f_{3}(k)=\frac{1}{{k^{\prime}}^{2}} \Gamma, \quad f_{4}(k)=\frac{1}{k^{4}}\left(2{k^{\prime}}^{2}-\left(1+k^{\prime 2}\right) \Gamma\right)$,

$f_{5}(k)=\Gamma$ $f_{6}(k)=\frac{1}{3}\left(\left(2-k^{2}\right) \Gamma-2 k^{\prime 2}\right)$.

\section{A.3 Solutions of sn-type}

In this section, we specialize the above expressions for the $f_{j}$ to solutions of sn-type (solutions of the form given in (6)). $L=4 K(k), k^{\prime}=\sqrt{1-k^{2}}$ and $\Gamma=E(k) / K(k)$.

$$
\begin{array}{rlrl}
f_{1}(k) & =\frac{1}{k^{\prime}}\left(k^{\prime 2}-2 \Gamma\right), & f_{2}(k) & =\frac{1}{k^{2} k^{\prime}}\left(k^{\prime 2}-\left(1+k^{2}\right) \Gamma\right), \\
f_{3}(k) & =\frac{1}{k^{2}}(1-\Gamma), & f_{4}(k)=k^{2}\left(\frac{1}{k^{\prime}}\left(k^{\prime 2}-2 \Gamma\right)-\frac{1}{k^{2} k^{\prime 2}}\left(\Gamma-k^{\prime 2}\right)\right), \\
f_{5}(k) & =1-\Gamma, & f_{6}(k)=\frac{1}{3}\left(\left(1+k^{2}\right) \Gamma-k^{\prime 2}\right) .
\end{array}
$$

\section{B Numerical parameters}

The numerical parameters used to create Figure 3 a are $N=16, N_{\rho}=800$ and $N_{\mu}=20$.

Table B.1 contains the numerical parameters used to create Figures 4-9. The parameters $N_{\rho}$ and $N_{\mu}$ were chosen so that the points in the spectra were sufficiently dense. The parameter $N$ was chosen well above what was necessary in order to ensure the numerical method had converged.

\section{References}

[1] V.A. Aleshkevich, A.A. Egorov, Y.V. Kartashov, V.A. Vysloukh, and A.S. Zelenina. Stability of spatiotemporal cnoidal waves in cubic nonlinear media. Phys Rev E, 67:066605, 2003. 
Table B.1

\begin{tabular}{|c|c|c|c||c|c|c|c|}
\hline \multicolumn{4}{|c||}{$\boldsymbol{\beta}>\mathbf{0}$} & \multicolumn{4}{c|}{$\boldsymbol{\beta}<\mathbf{0}$} \\
\hline$k$ & $N$ & $N_{\rho}$ & $N_{\mu}$ & $k$ & $N$ & $N_{\rho}$ & $N_{\mu}$ \\
\hline 0.2 & 20 & 400 & 100 & 0.2 & 20 & 800 & 100 \\
0.5 & 20 & 400 & 100 & 0.5 & 20 & 800 & 100 \\
0.9 & 50 & 400 & 100 & 0.9 & 50 & 800 & 100 \\
0.9999 & 100 & 400 & 100 & 0.9999 & 100 & 800 & 100 \\
\hline
\end{tabular}

Numerical parameters used to create Figures 4-9.

[2] D.J. Benney and G.J. Roskes. Wave instabilities. Stud Appl Math, 48(377):377-385, 1969.

[3] P.F. Byrd and M.D. Friedman. Handbook of Elliptic Integrals for Engineers and Physicists. Springer-Verlag, 1954.

[4] J.D. Carter and H. Segur. Instabilities in the two-dimensional cubic nonlinear Schrödinger equation. Phys Rev E, 68:045601, 2003.

[5] A. Davey and K. Stewartson. On three-dimensional packets of surface waves. Proc Royal Soc London Ser A, 338:101-110, 1974.

[6] B. Deconinck and J.N. Kutz. Computing spectra of linear operators using Hill's method. Submitted for publication, 2005.

[7] B. Deconinck, D. E. Pelinovsky, and J.D. Carter. Transverse instabilities of deep-water solitary waves. Submitted for publication, 2005.

[8] E.P. Gross. Hydrodynamics of a superfluid condensate. J Mathematical Phys, 4(2), February 1963.

[9] R.B. Guenther and J.W. Lee. Partial Differential Equations of Mathematical Physics and Integral Equations. Prentice Hall, New Jersey, 1988.

[10] E. Infeld and J. Ziemkiewicz. Stability of complex solutions of the nonlinear Schrödinger equation. Acta Phys Pol, A59(3):255, 1981.

[11] C.G. Jacobi. Fundamenta nova theoriae functionum ellipticarum. Königsberg, 1829.

[12] Y.V. Kartashov, V.A. Aleshkevich, V.A. Vysloukh, A.A. Egorov, and A.S. Zelenina. Transverse modulational instability of $(2+1)$-dimensional cnoidal waves in media with cubic nonlinearity. Phys Rev E, 67:036613, 2003.

[13] Y.V. Kartashov, V.A. Aleshkevich, V.A. Vysloukh, A.A. Egorov, and A.S. Zelenina. Transverse modulational instability of $(2+1)$-dimensional cnoidal waves in media with cubic nonlinearity. $J$ Opt Soc Am B, 20(6):1273-1284, 2003.

[14] T. Kato. Perturbation Theory for Linear Operators. Springer-Verlag, Berlin, 1966.

[15] Y.S. Kivshar and D.E. Pelinovsky. Self-focusing and transverse instabilities of solitary waves. Phys Rep, 331:117-195, 2000.

[16] E.A. Kuznetsov, A.M. Rubenchik, and V.E. Zakharov. Soliton stability in plasmas and hydrodynamics. Phys Rep, 142:103, 1986. 
[17] W. Magnus and S. Winkler. Hill's equation. Dover, New York, 1979.

[18] S.V. Manakov. On the theory of two-dimensional stationary self-focusing of electromagnetic waves. Sov Phys JETP, 38:248-253, 1974.

[19] D.U. Martin, H.C. Yuen, and P.G. Saffman. Stability of plane wave solutions of the two-space-dimensional nonlinear Schrödinger equation. Wave Motion, 2:215-229, 1980.

[20] H.L. Pecseli. Solitons and weakly nonlinear waves in plasmas. IEEE Trans Plasma Sci, 13(2):53-86, 1985.

[21] L.P. Pitaevskii. Vortex lines in an imperfect Bose gas. Sov Phys JETP, 13(2), 1961.

[22] K. Rypdal and J.J. Rasmussen. Stability of solitary structures in the nonlinear Schrödinger equation. Phys Scripta, 40:192, 1989.

[23] P.L. Sulem and C. Sulem. Nonlinear Schrödinger Equations: Self-focusing and Wave Collapse. Springer, New York, 1999.

[24] V.E. Zakharov and A.M. Rubenchik. Instability of waveguides and solitons in nonlinear media. Sov Phys JETP, 38(3):494-500, 1974. 